\title{
Role of Diffusion Tensor Imaging and Motor Functional MRI in Preoperative Assessment of Brain Tumors
}

\author{
Sara Essam Hasby ${ }^{1,}$, , Al-Shaimaa Zakaria Elshahawy ${ }^{1}$, Mohamed Amin Mohamed ${ }^{1}$, \\ Ali Ibraheem Seif Eldean ${ }^{2}$, Mohammed Fathy Dawoud ${ }^{1}$ \\ ${ }^{1}$ Department of Radiology, Faculty of Medicine, Tanta University, Tanta, Egypt \\ ${ }^{2}$ Department of Neurosurgery, Faculty of Medicine, Tanta University, Tanta, Egypt
}

Email address:

saraessam201420@yahoo.com (Sara E. H.), Shaymaazakaria512@gmail.com (Al-Shaimaa Z. E.), mohamin73rad@yahoo.com (Mohamed A. M.), ali.eldeen@med.tanta.edu.eg (Ali I. S. E.),

mohamed.dawood@med.tanta.edu.eg (Mohammed F. D.)

${ }^{*}$ Corresponding author

\section{To cite this article:}

Sara Essam Hasby, Al-Shaimaa Zakaria Elshahawy, Mohamed Amin Mohamed, Ali Ibraheem Seif Eldean, Mohammed Fathy Dawoud. Role of Diffusion Tensor Imaging and Motor Functional MRI in Preoperative Assessment of Brain Tumors. International Journal of Medical Imaging. Vol. 9, No. 1, 2021, pp. 16-28. doi: 10.11648/j.jimi.20210901.12

Received: December 20, 2020; Accepted: December 30, 2020; Published: January 12, 2021

\begin{abstract}
Diffusion tensor imaging is a non-invasive magnetic resonance imaging technique that describe the orientation and location of white matter tracts in vivo, it guides the surgeon regarding the relationship of the intra-axial tumor to adjacent white matter tracts in different planes. Routine pre-surgical FMRI allows for assessment of the relationship between brain tumor and motor cortex to facilitate function-preserving surgery and reduce postoperative morbidity. The purpose of this study is to evaluate role of diffusion tensor magnetic resonance imaging, fiber tractography and motor functional MRI in assessment of white matter tracts affected by the tumor, and to utilize this information in guiding surgeons. Fifty patients with different types of brain tumors were enrolled in this study. Fiber tracts involvement were classified into four categories (edema, displacement, infiltration and disruption), comparison between benign and malignant tumors groups as regards to the patterns of fiber tracts involvement were done. The prevalence of edema and displacement was higher among the benign group while prevalence of infiltration and disruption was higher among the malignant group. Localization of the primary motor hand area with functional MRI was done, The relation of it to the tumor was measured. Treatment plans before and after DTI \& functional MR imaging differed in 35 patients as regard to surgical approach and in 17 patients as regard to modification of extent of resection with significant relation. Diffusion tensor imaging DTI represents recent important progress in the field of diagnostic imaging. It is the only technique that is able to visualize in vivo white-matter tracts so that it is of great help in preoperative neurosurgical planning.
\end{abstract}

Keywords: Diffusion Tensor Imaging, Fiber Tractography, Motor Functional MRI, Brain Tumors

\section{Introduction}

The pre-operative surgical planning and the assessment of the surrounding white matter (WM) tracts is a very important step in patients with brain tumors as it allows the complete resection of tumor, and avoiding recurrence and loss of vital brain functions such as motor, sensory, language at the same time. Conventional MRI (cMRI) in a routine that demonstrates the lesion without any accurate information about the tumor involvement and also about the integrity of the surrounding white matter tracts [1].

Diffusion-tensor imaging and fiber tractography are the only available tool currently which displays the disruption of the white matter tracts by measuring the changes of fractional anisotropy (FA) and can assess the main fibers tracts in patients with malignant brain tumors. therefore, it is very important to localize the white matter tracts accurately in preoperative surgical planning and in making the decision of whether to operate or not [2].

Diffusion-tensor imaging (DTI) is mainly based on the random diffusion of water molecules in each voxel. It 
provides directional information about fiber tracts and also provides three maps: the directionally encoded color-coded map (DEC), apparent diffusion coefficient (ADC) map, and fractional anisotropy (FA) map [3].

\section{Patients and Methods}

The study aimed at exploring the value of Diffusion Tensor MR Imaging and motor functional imaging in the preoperative assessment of brain tumors. The study included 50 patients with different pathological types of brain tumors.

\subsection{Study Population}

This study was conducted in MRI unit of Radio-diagnosis department, Faculty of medicine, Tanta University.

\subsection{Inclusion Criteria}

Patients of any age with evidence of resectable brain tumors.

\subsection{Exclusion Criteria}

1) Patients known to have contraindications for MRI, e.g. an implanted magnetizable device, pacemakers or claustrophobia.

2) Patients with contraindication to contrast; Renal failure with GFR less than $30 \mathrm{ml} / \mathrm{min}$.

3) Patients with irresectable brain tumors.

\subsection{Methods}

\subsubsection{Each Patient Included in the Study Was Subjected to}

1) Full history taking,

2) General examination

3) Neurological examination:

\subsubsection{Patient Preparation}

1) Obtaining an informed consent by the patient, patient's parents or care givers before MRI examination.

2) Measuring patient body weight for calculation of amount of contrast media and sedative material if indicated.

3) Fasting for 6-8 hours and checking serum creatinine before Gadolinium administration.

\subsubsection{MRI Technique}

1) Technique was performed using a standard 1.5 Tesla unit (GE HealthCare, Sigma HDX., W).

2) A standard head coil was used.

\subsubsection{Magnetic Resonance Imaging Protocol Include}

1) Pre \& post contrast High-resolution 3D T1-weighted spoiled gradient echo pulse sequence was acquired; (TR/TE/TI, 9.7/4.6/400 ms, flip angle $(\theta)=35^{\circ}, 124$ slices $0.8 \mathrm{~mm}$ thick, $208 \times 170$ matrix, field of view (FOV) $23 \mathrm{~cm} 260$ contiguous sections, acquisition time $5.25 \mathrm{~min})$

2) Diffusion Tensor imaging, which consisted of a single shot, spin-echo echoplanar sequence in 40 encoding directions and a diffusion weighting factor of $800 \mathrm{~s} / \mathrm{mm} 2$. (TR) 10951, TE 67 , matrix 128 x128, FOV 224 X $224 \mathrm{~mm}$, number of excitations 2, slice thickness: 2.0/00 and flip angle 90 (degrees).

3) Functional Magnetic Resonance Imaging ( BOLD technique) (motor functional magnetic resonance imaging paradigm using block design (alternating action and rest tasks) were used to explore motor cortex in selected cases (patients with symptoms involving tremors, epilepsy, abnormal movements and disturbed conscious level were excluded ).

4) For functional MRI: a T2*-weighted gradient-echo echo-planar imaging (EPI) sequence was used(TR/TE, 2540/45 ms;matrix, 64x64; FOV, $224 \mathrm{~mm}$; section thickness, $5 \mathrm{~mm}$; 50 contiguous sections) to obtain blood oxygen level dependent (BOLD). Total fMRI scan time was approximately 5 minutes and 12 seconds.

\subsubsection{Data Processing and Analysis}

All the diffusion-tensor and functional MRI images were transferred to the workstation (Advantage workstation 4.7). Images were post-processed using the GE software devised for tractography. The maps obtained were:

1) FA maps.

2) directionally-encoded color FA maps.

3) 3D fiber tractography maps.

The direction and anatomy of the tracts are seen in the directionally-encoded FA maps, where a specific color is assigned to tracts running in the three orthogonal planes:

1) Red is for right to left tracts.

2) Green for anteroposterior tracts.

3) Blue for craniocaudal tracts.

A 3D display of tracts was created. For creating 3D fiber tracts, a ROI (or seed) was drawn (placed) along the course of the tract in the (axial, sagittal or coronal) color encoded FA map in single or consecutive sections. The software then automatically traces the assigned tract and presents it in a 3D manner.

Regions of interest (ROIs) were drawn within identifiable white matter tracts affected by tumor, avoiding grossly cystic and necrotic regions, known fiber crossings and gray matter.

Color-coded DTI maps were analyzed, both subjectively by visual comparison and quantitatively by comparing the FA values with the contra lateral normal side and, this was followed by tractography of individual tracts, the location of each tract and its hue on directional color maps were classified as normal or abnormal, based on comparison to the homologous tracts in the contralateral hemisphere which were unaffected by tumor.

\subsubsection{Fiber Tracts Classification}

Fiber tracts involvement was classified into 4 categories: Deviated (Displaced), Edematous, Infiltrated and Destructed (Disrupted).

A. Displacement:

1) Maintained normal anisotropy relative to the contralateral tract.

2) Situated in abnormal location or presented by abnormal 
orientation.

3) Directional changes reflected by abnormal hue on directional maps.

4) Identifiable intact fiber tracts.

B. Invasion:

1) Reduced anisotropy relative to the contra-lateral tract.

2) Still identifiable fiber tract.

3) Normal orientation on directional color maps.

C. Disruption:

1) Markedly reduced anisotropy relative to the contralateral tract.

2) The fiber tract is unidentifiable on directional color maps.

E. Edema:

1) Markedly reduced anisotropy relative to the contralateral tracts.

2) Normal orientation on directional color maps.

3) Located in abnormal T2 WIs signal intensity area.

\subsubsection{Motor Functional MRI Task}

Before functional imaging, all cases and controls were given a single training session lasting about 20minutes to habituate them with the action of paradigm. Subjects lay supine in the scanner with supporting pads under their ankles and knees permitting free movement at the ankle only. Both arms were also supported to permit only movements of fingers. The experiment was conducted using a block design of alternating task and rest periods, each lasting $30 \mathrm{~s}$, the basic task consisted of 10 blocks. Instructions were reflected on a mirror in front of the patient's eyes from a screen showing pictures of body parts (legs/arms), arrow of laterality and animation of action and rest. We used fMRI to test the activation pattern produced during physical attempts to move or during mental imagery of moving the limb of the contra lateral side to the brain lesion. Healthy controls were asked to carry out the same task.

\subsection{8. fMRI Data Processing and Analysis}

Data processing and analysis of fMRI data were completed on GE workstation (ADW 4.7). TheT2*-weighted functional images were readjusted, block-time corrected according to the paradigm, functional and 3D T1 WI anatomical images were then fused. Statistical parametric maps were calculated by using the general linear model by modeling the "active" and "rest" conditions as a boxcar function elaborated with the hemodynamic response function.

Paradigm functional MRI and 3D T1 WI anatomical images fused revealed site of primary motor cortex of the left hand of a normal control patient.

\subsubsection{Statistical Analysis}

Statistical presentation and analysis of the present study was conducted, using the mean, standard deviation, Paired ttest, Chi-square tests by SPSS V17.

\section{Results}

This study included 50 patients with brain tumors, 33 of them were males and this represents $66 \%$ of the cases and the remaining were females \&representing $34 \%$ of them. Their ages ranged between 2 and 69 years old with mean of about 52.6 year. The study also included 10 age matched healthy control group who were referred for other causes and their MRIs of the brain were normal. will show the sex \& age distribution of the studied 50 cases with brain tumors.

The patients were presented with different clinical manifestations, among the studied 50 cases; 15 patients presented mainly with headache, 10 cases with disturbed conscious level, 12 cases with hemiparesis and 8 cases with convulsions 4 cases with repeated syncopal attacks and 1 case with aphasia.

The study included different pathological types of brain tumors, most common diagnosis were gliomas with their different grades.

The lesions included in this study consisted of: (Table 1)

Table 1. Number and percentage of different pathological types of cases with brain tumors: $n=$ number, $\%=$ percentage.

\begin{tabular}{lll}
\hline Diagnosis & N & \% \\
\hline Glioblastoma Multiform & 22 & 44 \\
Astrocytoma & 5 & 10 \\
Lymphoma & 4 & 8 \\
Low Grade Glioma & 3 & 6 \\
Meningioma & 3 & 6 \\
Trigeminal Shwanoma & 3 & 6 \\
Metastatic deposits & 2 & 4 \\
Ependymoma & 2 & 4 \\
Gliosarcoma & 1 & 2 \\
DNET & 1 & 2 \\
Oligodendroglioma & 1 & 2 \\
Ganglioglioma & 1 & 2 \\
Hemangiopericytoma & 1 & 2 \\
Central neurocytoma & 1 & 2 \\
Total & 50 & 100 \\
\hline
\end{tabular}

The relation between nature of the lesions and pattern of tract affection:

Fiber tracts involvement were classified into four categories (edema, displacement, infiltration and disruption), comparison between benign and malignant tumors groups as regards to the patterns of fiber tracts involvement.

\section{A: Edema}

Table 2. The comparison between benign and malignant tumors as regard to the pattern of edema: (n: number, \%: percentage).

\begin{tabular}{|c|c|c|c|c|c|}
\hline \multirow{2}{*}{ Edema } & \multicolumn{2}{|c|}{ malignant } & \multicolumn{2}{|c|}{ Benign } & \multirow{2}{*}{$\begin{array}{l}\text { Chi Square } \\
\text { Test }\end{array}$} \\
\hline & $\mathbf{N}$ & $\%$ & $\mathbf{N}$ & $\%$ & \\
\hline Present & 20 & $7.5 \%$ & 0 & $0 \%$ & P-value \\
\hline Not present & 244 & $92.5 \%$ & 36 & $100 \%$ & 0.08 \\
\hline
\end{tabular}

P: p-value for Chi-square test for comparison between benign and malignant tumors as regard to the pattern of edema.

*: Statiscally significant at $\mathrm{p}<0.05$.

This table showed that prevalence of edema was higher among the malignant group in comparison to the benign group but with no significant difference in between by using the chi-square test.

B: Displacement 
Table 3. The comparison between benign and malignant tumors as regard to the pattern of displacement: (n: number, \%: percentage).

\begin{tabular}{llllll}
\hline \multirow{2}{*}{ Displacement } & \multicolumn{2}{l}{ malignant } & \multicolumn{2}{l}{ Benign } & Chi Square \\
\cline { 2 - 5 } & $\mathbf{N}$ & $\mathbf{\%}$ & $\mathbf{N}$ & $\mathbf{\%}$ & Test \\
\hline Present & 45 & $18 \%$ & 14 & $38 \%$ & P-value \\
Not present & 219 & $82 \%$ & 22 & $61 \%$ & $<0.001^{*}$ \\
\hline
\end{tabular}

P: p-value for Chi-square test for comparison between benign and malignant tumors as regard to the pattern of displacement.

*: Statiscally significant at $\mathrm{p}<0.05$.

This table showed that the prevalence of displacement was higher among the benign group in comparison to the malignant group with significant difference in between by using the chi-square test.

\section{C: Infiltration}

Table 4. The comparison between benign and malignant tumors as regard to the pattern of infiltration: (n: number,\%: percentage)

\begin{tabular}{llllll}
\hline \multirow{2}{*}{ Infiltration } & \multicolumn{2}{l}{ malignant } & \multicolumn{2}{l}{ Benign } & Chi Square \\
\cline { 2 - 5 } & $\mathbf{N}$ & $\mathbf{\%}$ & $\mathbf{N}$ & $\mathbf{\%}$ & Test \\
\hline Present & 51 & $19.3 \%$ & 0 & $0 \%$ & P-value \\
Not present & 213 & $80.7 \%$ & 36 & $100 \%$ & $<0.001^{*}$ \\
\hline
\end{tabular}

P: p-value for Chi-square test for comparison between benign and malignant tumors as regard to the pattern of infiltration.

*: Statiscally significant at $\mathrm{p}<0.05$.

This table showed that prevalence of infiltration was higher among the malignant group in comparison to the benign group with significant difference in between by using the chi-square test.

D: Disruption

Table 5. The comparison between benign and malignant tumors as regard to the pattern of disruption:(n: number,\%: percentage).

\begin{tabular}{llllll}
\hline \multirow{2}{*}{ Disruption } & \multicolumn{2}{l}{ Malignant } & \multicolumn{2}{l}{ Benign } & Chi Square \\
\cline { 2 - 5 } & $\mathbf{N}$ & $\mathbf{\%}$ & $\mathbf{N}$ & $\mathbf{\%}$ & Test \\
\hline Present & 40 & $15.2 \%$ & 0 & $0 \%$ & P-value \\
Not present & 224 & $84.8 \%$ & 36 & $100 \%$ & $<0.001^{*}$ \\
\hline
\end{tabular}

P: p-value for Chi-square test for comparison between benign and malignant tumors as regard to the pattern of disruption.

*: Statiscally significant at $\mathrm{p}<0.05$.

The distance relation between primary motor hand area and tumor location

In this study 45 patients with different brain tumors were assessed by motor functional MRI, although 12 of the patients suffered from tumor-associated pareses, localization of the motor hand area was still possible in all but 5 cases, they were excluded as they were non cooperative patients.

Localization of the primary motor hand area with functional MRI was done; its relation to the tumor was classified according to the distance between into:

(a) Motor area was more than $2 \mathrm{~cm}$ away from the tumor.

(b) Motor area was 1-2 cm away from the tumor.

(c) Motor area was less than $1 \mathrm{~cm}$ away from the tumor.

(d) Motor area was zero distance from the tumor border or lies within the tumor and/or infiltrated by it.

Motor area was more than $2 \mathrm{~cm}$ away from the tumor that was reported in 19 patients $(42.22 \%)$, (b) motor area was $1-2$ $\mathrm{cm}$ away from the tumor and (c) motor area was less than 1 $\mathrm{cm}$ away from the tumor each of the were reported in 5 patients $(11.11 \%)$, (d) motor area was zero distance from the tumor border or lies within the tumor and/or infiltrated by it was reported in 16 patients $(35.55 \%)$.

Table 6. Distance relation between primary hand motor area and the tumor location.

\begin{tabular}{lllll}
\hline $\begin{array}{l}\text { Primary } \\
\text { hand motor } \\
\text { area }\end{array}$ & $\begin{array}{l}\text { More than 2 } \\
\text { cm away } \\
\text { from tumor }\end{array}$ & $\begin{array}{l}\text { From1to 2 } \\
\text { cm away } \\
\text { from tumor }\end{array}$ & $\begin{array}{l}\text { Less than } \\
\mathbf{1} \text { cm from } \\
\text { the tumor }\end{array}$ & $\begin{array}{l}\text { Zero distance } \\
\text { or with in the } \\
\text { tumor }\end{array}$ \\
\hline $\begin{array}{l}\text { Number } \\
\%\end{array}$ & 19 & 5 & 5 & 16 \\
\hline
\end{tabular}

Assessment of the impact of tractography and motor functional MRI on the neurosurgical planning:

This study was a methodology to assess the practical impact that the DTI and motor functional MRI information had on surgical planning and procedure performance was used. The neurosurgeons were asked to assess, the type of surgical approach and the extent of surgical resection established with conventional MR images and with the same images integrated with the tractography and motor functional MRI.

Results obtained were tabled as yes or no for the two parameters (surgical approach and resection margins). When at least one of the two parameters was modified by the tractography and motor functional MRI data, the surgical procedure was considered to have been modified. (Table 7)

Table 7. Assessment of the impact of tractography and motor functional MRI on changes of the neurosurgical planning.

\begin{tabular}{llllll}
\hline $\begin{array}{l}\text { Change in } \\
\text { treatment } \\
\text { plans }\end{array}$ & \multicolumn{2}{l}{$\begin{array}{l}\text { Modification of } \\
\text { surgical approach }\end{array}$} & $\begin{array}{l}\text { Modification of } \\
\text { extent of resection }\end{array}$ & $\begin{array}{l}\text { Chi Square } \\
\text { Test }\end{array}$ \\
\cline { 2 - 5 } & $\mathbf{N}$ & $\%$ & $\mathbf{N}$ & $\%$ & \\
\hline Yes & 10 & $22.2 \%$ & 28 & $62.2 \%$ & P-value \\
No & 35 & $77.8 \%$ & 17 & $37.8 \%$ & $\mathrm{p}<0.001^{*}$ \\
\hline
\end{tabular}

P: p-value for Chi Square Test Assessment of the impact of tractography and motor functional MRI on changes of the neurosurgical planning. *: Statiscally significant at $\mathrm{p}<0.05$.

As regard to the assessment of the impact of tractography and motor functional MRI on changes of the neurosurgical planning we found that the treatment plans before and after DTI \& functional MR imaging differed in 10 patients as regard to surgical approach and in 28 patients as regard to modification of extent of resection with significant relation $(\mathrm{P}<.05)$.

\section{Cases}

\subsection{Case 1}

A 55 year old male patient with right parietal lobe Astrocytoma manifested clinically with left sided weakness. 


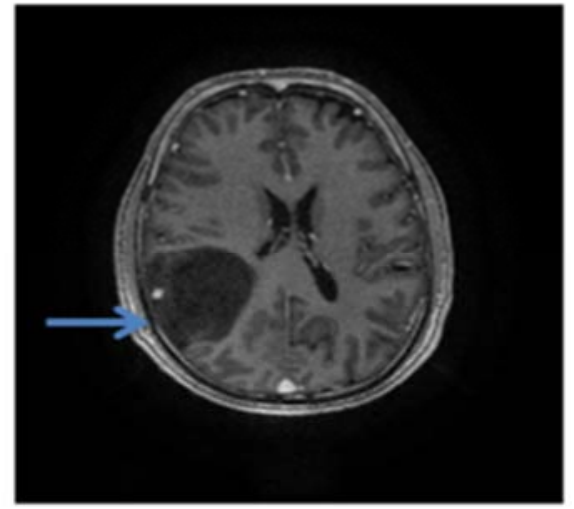

(A) Axial three dimension T1WI with contrast.

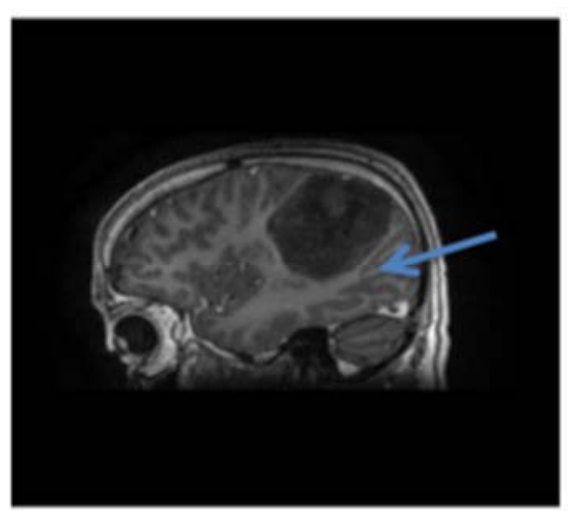

(B)S agittal three dimension T1WI with contrast.

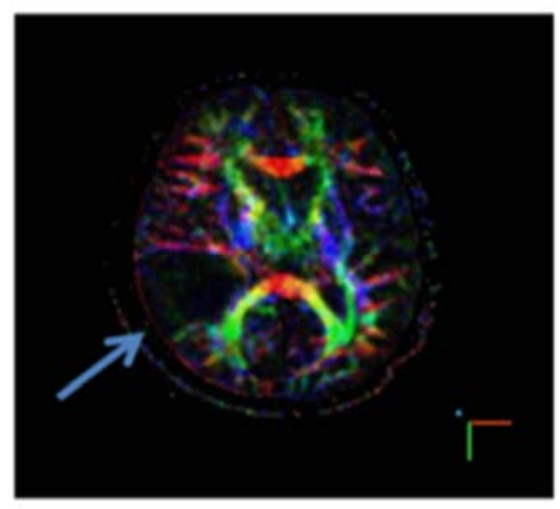

(C) Axial colour coded map

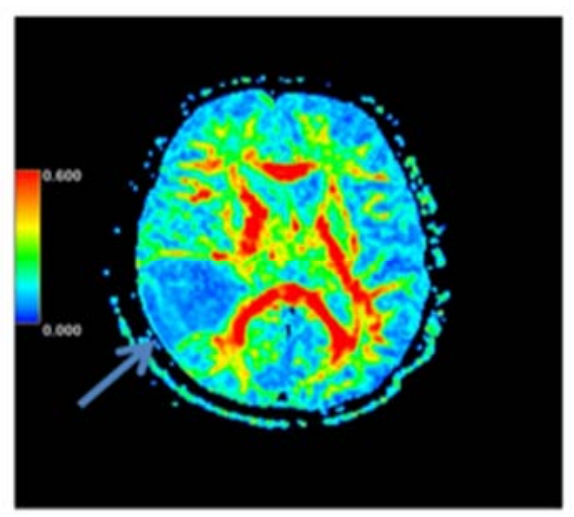

(D) Axial Fractional anisotropy map

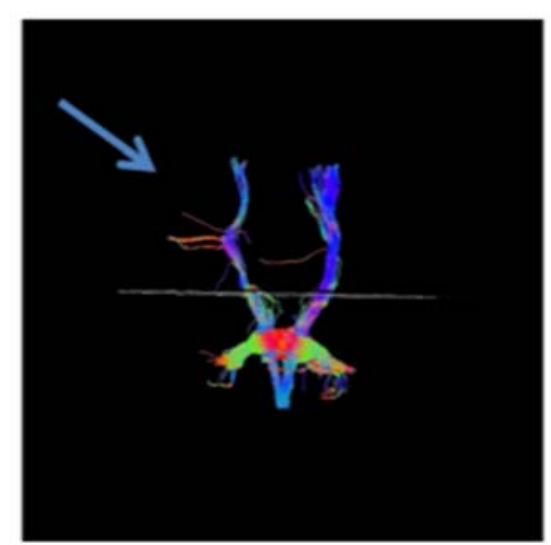

(E) 3D fiber tractography Corticospinal tract

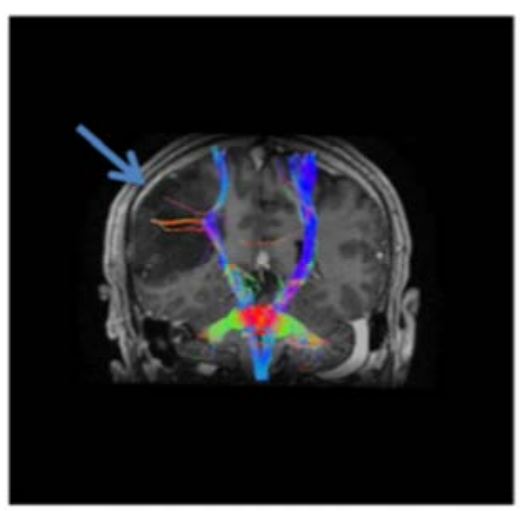

(F) 3D fiber tractography Corticospinal tract

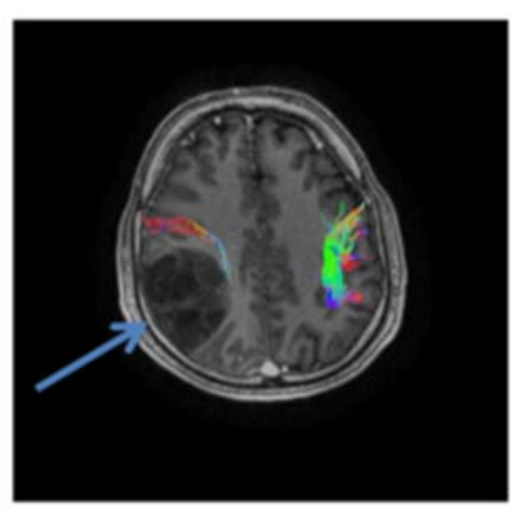

(G) 3D fiber tractography Superior Longtudinal Fasiculus

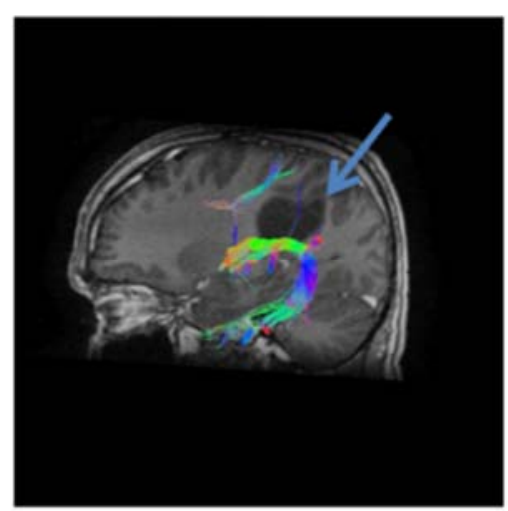

(H) 3D fiber tractography Superior Longtudinal Fasiculus 


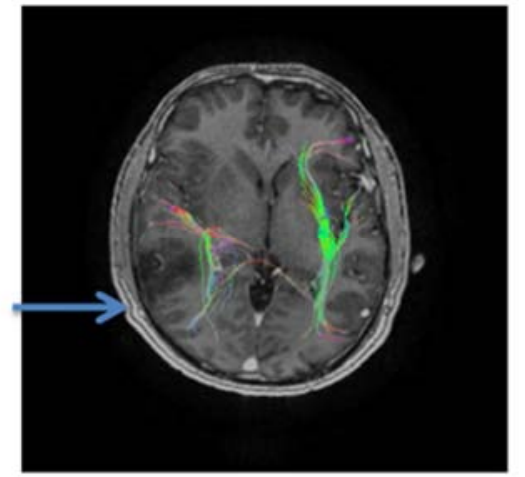

(I) 3D fiber tractography Inferior Longtudinal Fasiculus

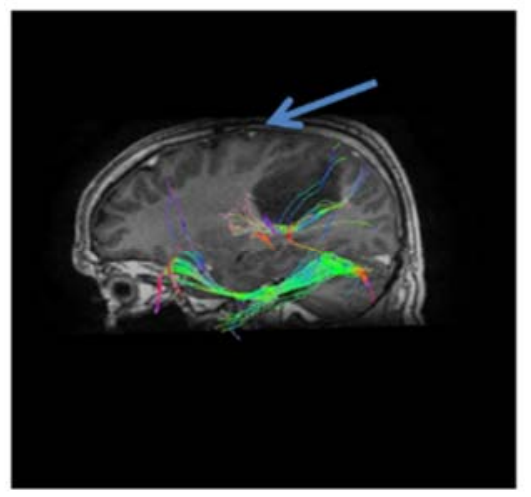

(J) 3D fiber tractography Inferior Longtudinal Fasiculus

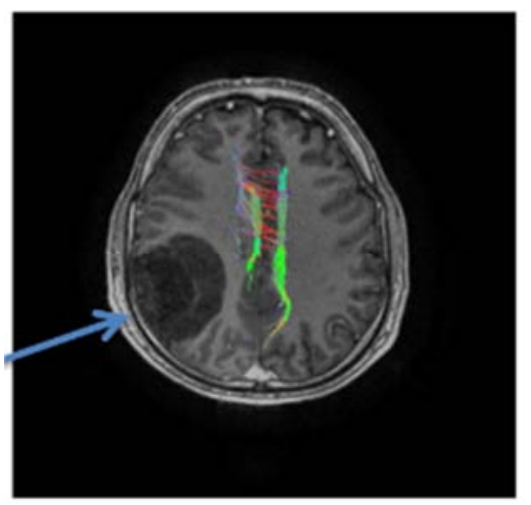

(K) 3D fiber tractography Cingulum

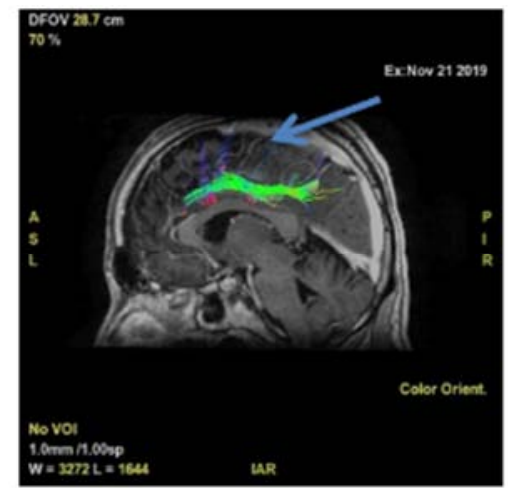

(L) 3D fiber tractography Cingulum

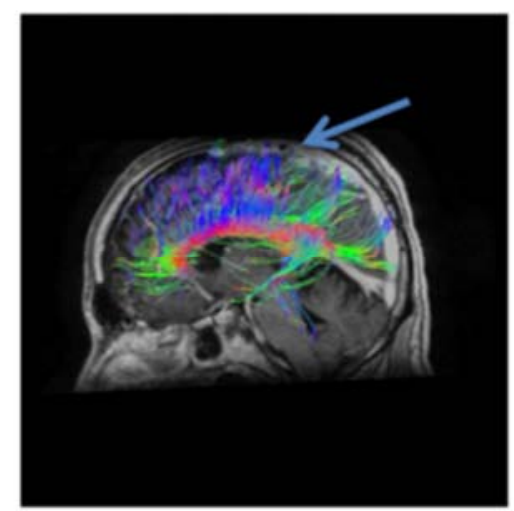

(M) 3D fiber tractography Corpus Callosum

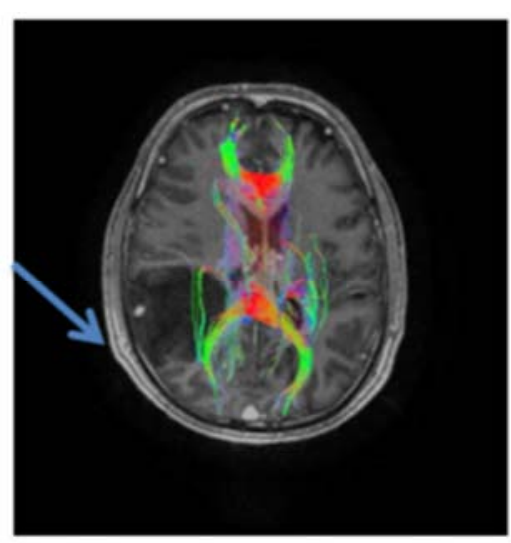

(N) 3D fiber tractography Corpus Callosum

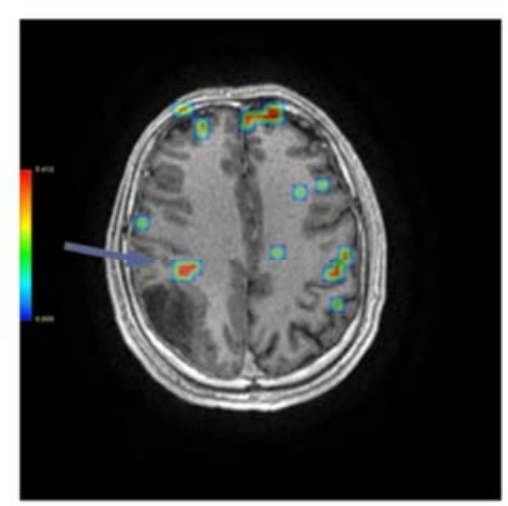

(O) Functional MRI primary motor area of the hand

Figure 1. $(A \& B)$ axial and sagittal $3 D T 1 W I$ with contrast showed evidence of large fairly defined right sided parietal lobe space occupying cystic lesion with small solid nodule that is showing homogenous enhancement after IV contrast injection, it is seen exerting mass effect in the form of effacement of ipsilateral cortical sulci and compressing the occipital horn of the right lateral ventricle. (C) axial color coded map showed affection of the colours corresponding to the site of the tumor which revealed affection of the corresponding fibers, (D) FA map revealed reduction of the FA parameters in the tracts related to the tumor $(E)$ to $(N)$ three dimension fiber tractography maps revealed infiltration of both cortico-spinal and Inferior Longitudinal Fasciculus tracts on the right side while destruction of the Superior Longitudinal Fasciculus tract and dispalceemnt of the cingulum tract are noted on the the affected right side. $(O)$ Multiplanar reconstruction of left hand activation map fused with $3 D T 1$ with contrast images localized primary motor area of the left hand that was dispalecd by the tumour mass with zero distance in between. 


\subsection{Case 2}

A female patient aged 28 years old with DNET manifested clinically with headache underwent MRI brain scan that revealed.

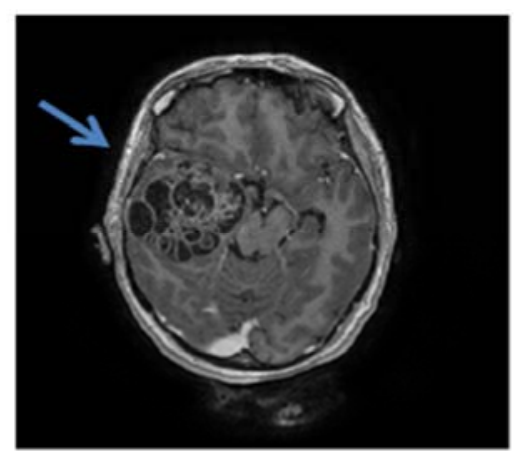

(A) Axial three dimension T1WI with contrast.

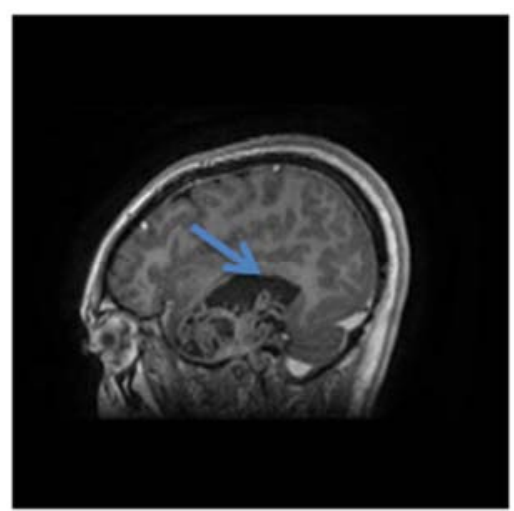

(B) Sagittal three dimension T1WI with contrast.

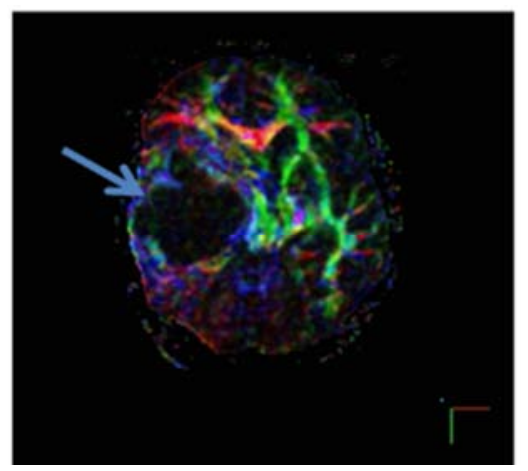

(C) Axial colour coded map.

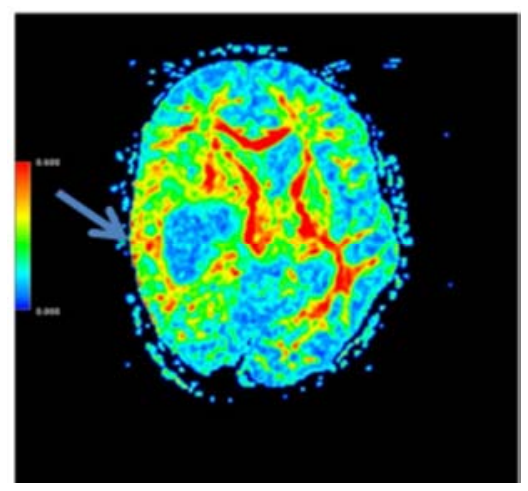

(D) Axial Fractional anisotropy map.

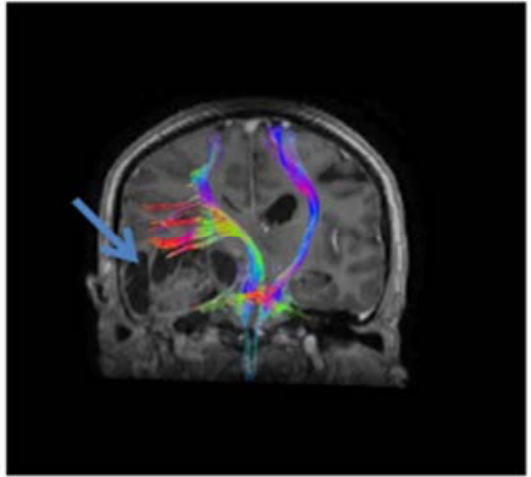

(E) 3D fiber tractography Corticospinal tract

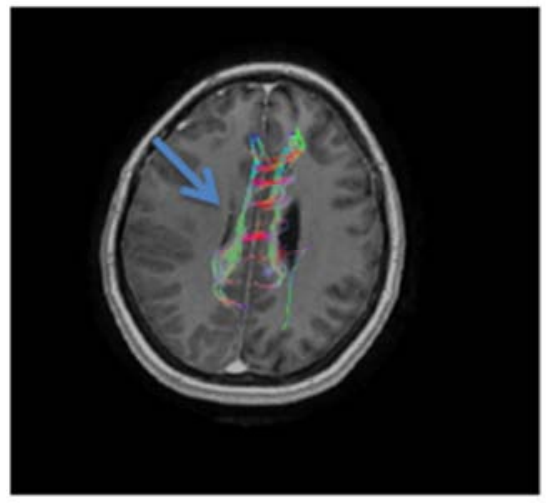

(F) 3D fiber tractography Cingulum

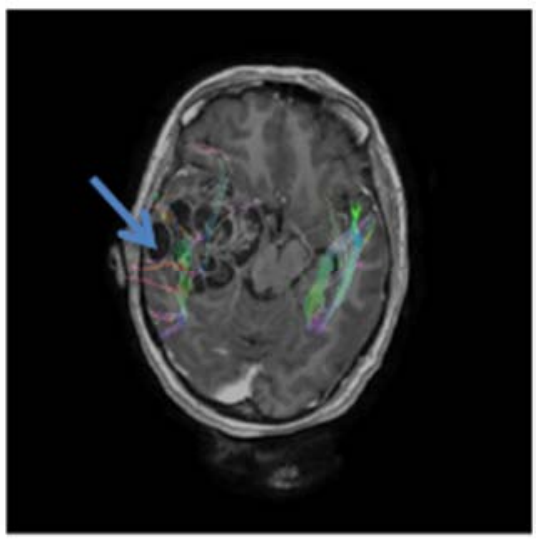

(G) 3D fiber tractography Superior Longtudinal Fasiculus

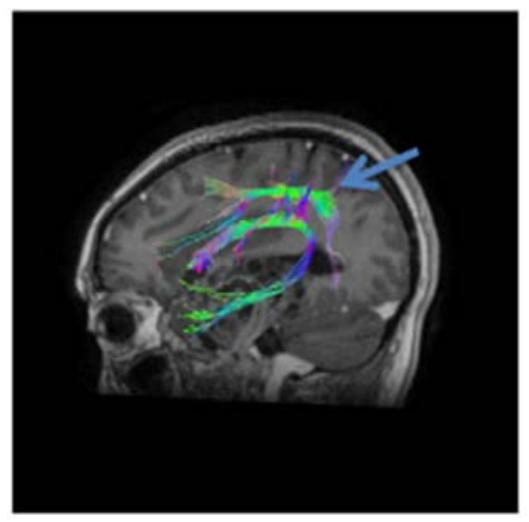

(H) 3D fiber tractography Superior Longtudinal Fasiculus 


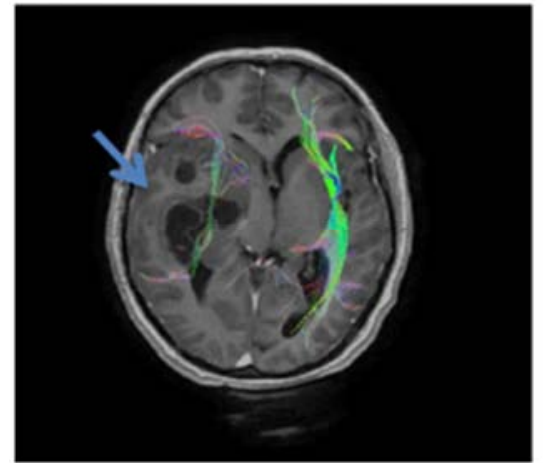

(I) 3D fiber tractography Inferior Longtudinal Fasiculus

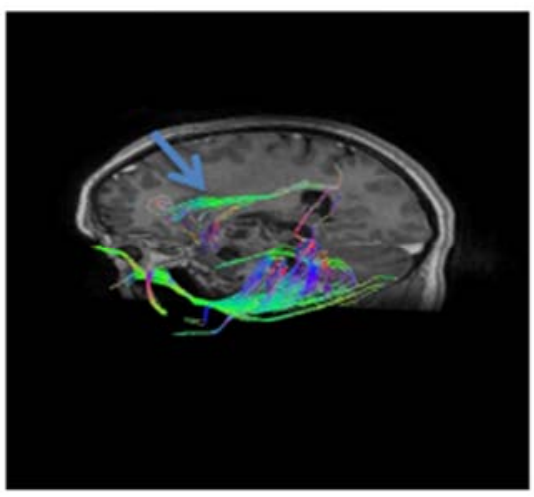

(J) 3D fiber tractography Inferior Longtudinal Fasiculus

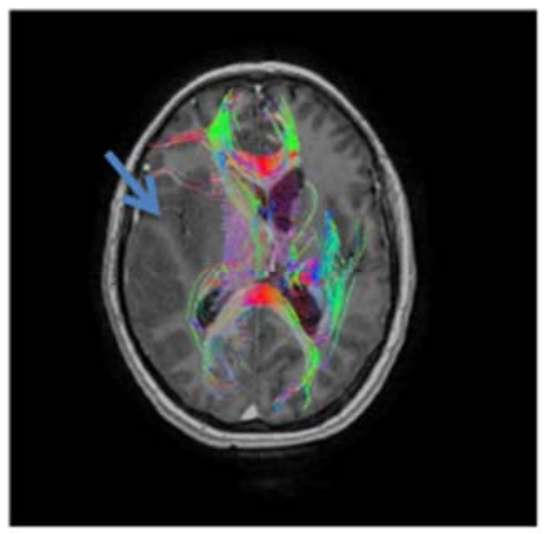

(K) 3D fiber tractography Corpus Callosum

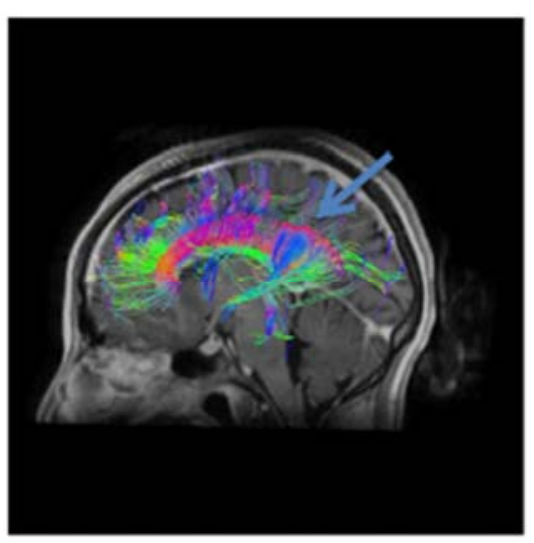

(L) 3D fiber tractography Corpus Callosum

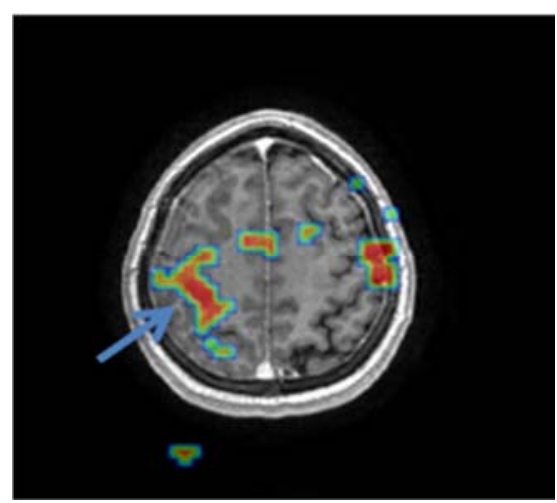

(M) Functional MRI primary motor area of the hand (axial).

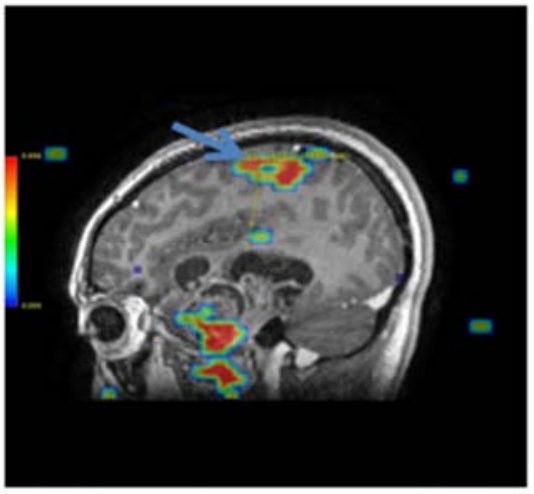

(N) Functional MRI primary motor area of the hand (sagittal).

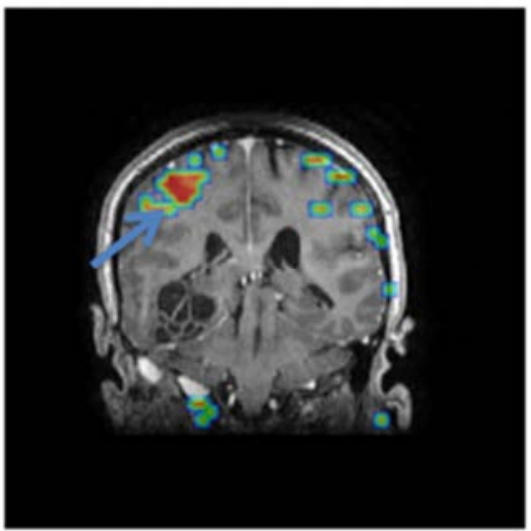

(O) Functional MRI primary motor area of the hand (cronal).

Figure 2. $(A \& B)$ axial and sagittal $3 D T 1 W I$ with contrast showed evidence of right tempero-parietal lobulated space occupying lesion displaying low signal intensity at T1WI with peripheral enhancement of its wall, the lesion exerting mass effect in the form of compression of the ipsilateral lateral ventricle and effacement of the ipsilateral cortical sulci with parafalcine herniation. (C) axial color coded map showed affection of the colors corresponding to the site of the lesion which revealed affection of the corresponding fibers, (D) FA map revealed reduction of the FA parameters in the tracts related to the tumors. (from $E$ to $M$ ) three dimension fiber tractography maps revealed infiltration of both Cortico-Spinal and Inferior Longitudinal Fasciculus tracts by the mass and deviated by its edema with displacement of the superior longitudinal fasciculus tract and Corpus Callosum tract on the affected right side in comparison to the normal side, normal fiber tracts of the Cingulum. (N,O \& $P$ ) multiplanar reconstruction of left hand activation map fused with $3 D$ T1 with contrast images localized the primary motor area of the left hand that was about $3.5 \mathrm{~cm}$ far from the tumour mass. 


\subsection{Case 3}

A female patient with known right sided meningioma aged 50 years old manifested clinically with headache

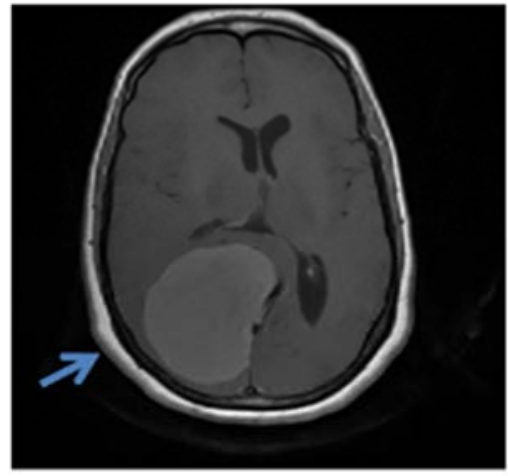

(A) Axial three dimension T1WI with contrast.

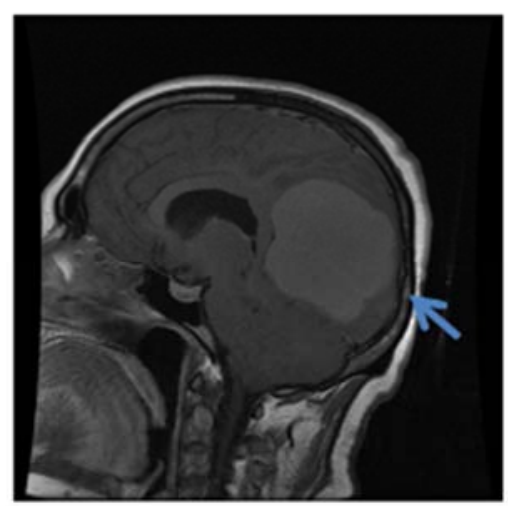

(B) Sagittal three dimension T1WI with contrast.

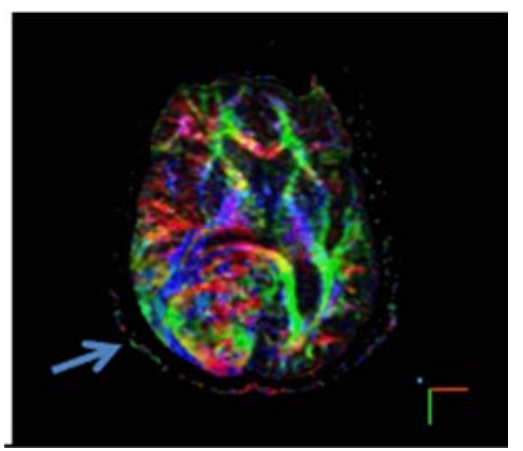

(C)Axial colour coded map.

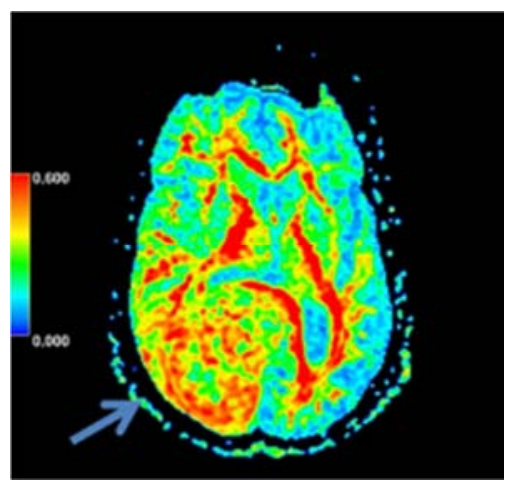

(D)Axial Fractional anisotropy map

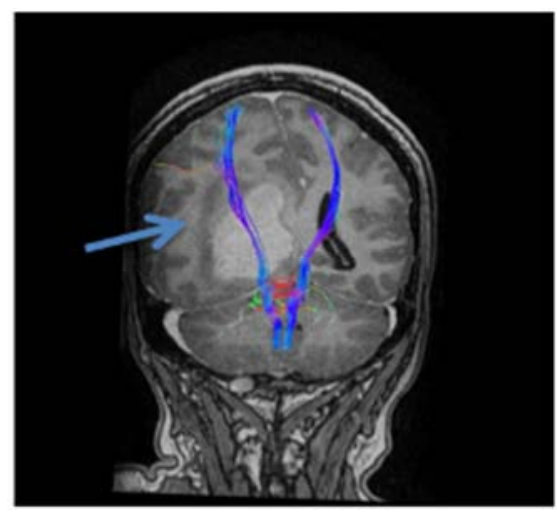

(E)3D fiber tractography Corticospinal tract

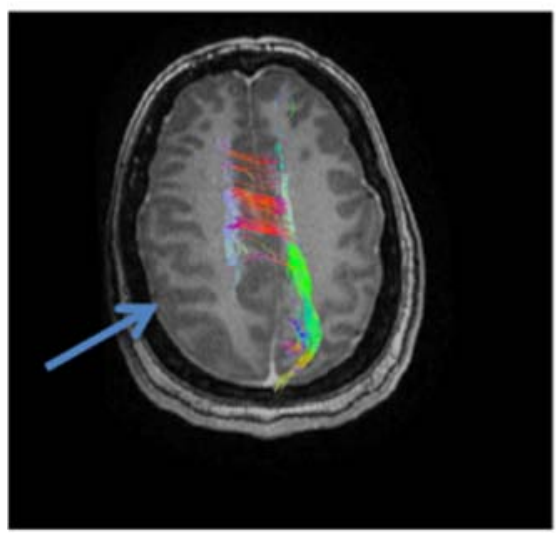

(F) 3D fiber tractography Cingulum

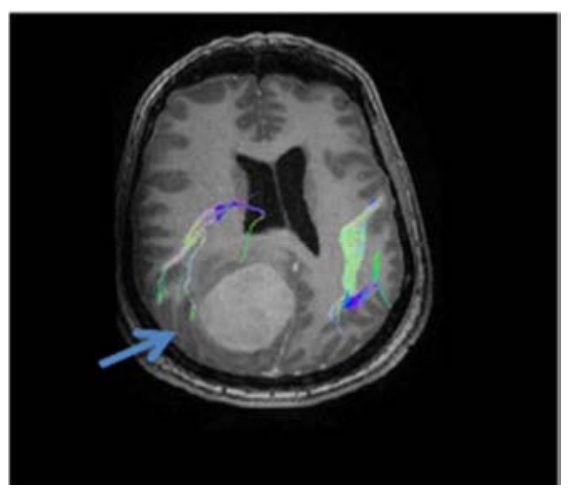

(G) 3D fiber tractography Superior Longtudinal Fasiculus

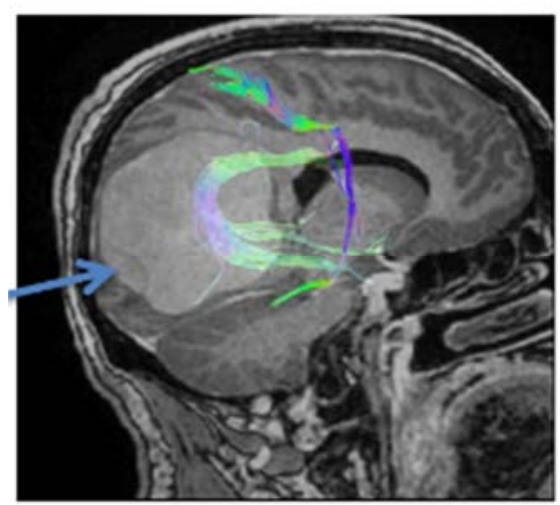

(H) 3D fiber tractography Superior Longtudinal Fasiculus 


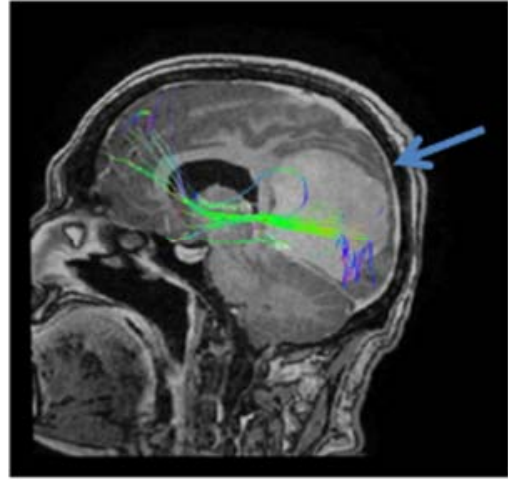

(I) 3D fiber tractography Inferior Longtudinal Fasiculus.

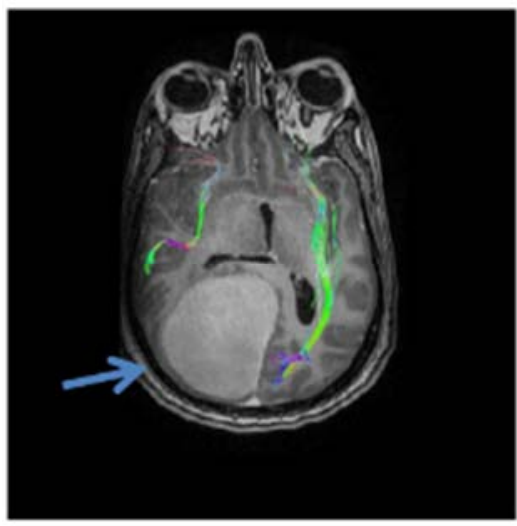

(J) 3D fiber tractography Inferior Longtudinal Fasiculus.

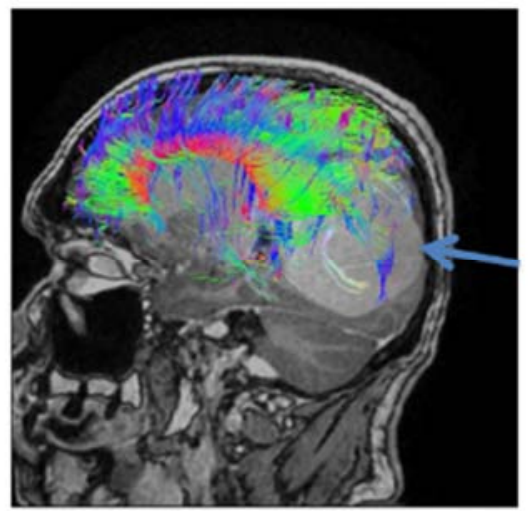

(K) 3D fiber tractography Corpus Callosum.

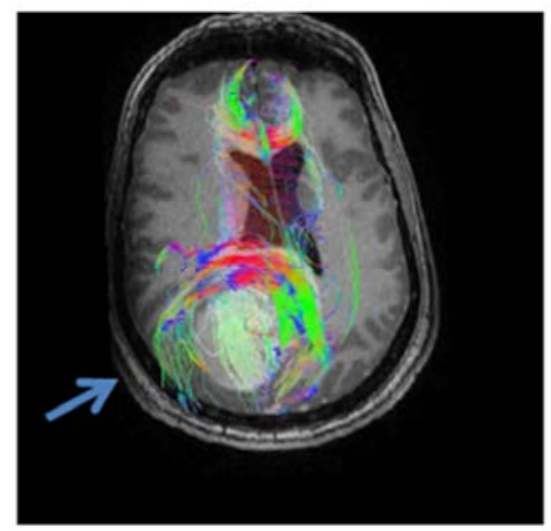

(L) 3D fiber tractography Corpus Callosum.

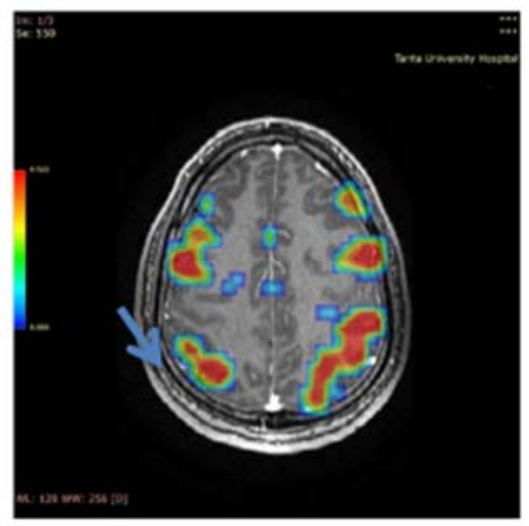

(M) Functional MRI primary motor area of the hand.

Figure 3. $(A \& B)$ axial and sagittal $3 D T 1 W I$ with contrast showed evidence of a well defined right sided parieto-occipital lobe extra-axial space occupying lesion with intense homogenous enhancement after IV contrast injection, exerting mass effect in the form of effacement of the ipsilateral cortical sulci and compression of the right lateral ventricle. (C) axial Colour coded map showed affection of the colours corresponding to the site of the lesion which revealed affection of the corresponding fibers \& (D) FA map revealed reduction of the FA parameters in the tracts related to the tumors (from $E$ to $L$ ) three dimension fiber tractography maps revealed displacement of the Superior and Inferior Longitudinal Fasciculus tracts and also displacement of the Corpus Callosum fiber tracts on the involved right side in comparison the contralateral healthy side, normal fiber tracts of the Cortico-Spinal tract and Cingulum. (M) Multiplanar reconstruction of left hand activation map fused with $3 D T 1$ with contrast images revealed bilateral representation of the primary motor area of the left hand that was away from the tumor surface.

\section{Discussion}

The traditional magnetic resonance imaging (MRI) scan can provide information about the intracranial structure, tumor size and location; however, it cannot reveal the relation of the tumor with the intracranial nerve fibers. After the development of MRI, diffusion tensor imaging (DTI) can provide non-invasive studies of the white matter fiber tracts. So nowadays DTI and fiber tractography can reflect the pathological state of the white matter tracts and the relation with adjacent tumors, so it can help the surgeon to design a suitable surgical plan prior to surgery [4].

In the current study, 50 patients with different pathological types of brain tumors were studied. 33 of the patients were males and this represents $66 \%$ of the cases and the remaining were females \&representing 34\% of them. This male predominance agreed with T. Sun [5] who found that differences in brain tumor rates and outcome are evident in males and females throughout the world and regardless of age.

The age of the studied patients ranged from 2 and 69 years with a mean of about 52.6 year, The majority of the studied cases were above 40 years. K. Farmanfarma [4] reported that The incidence of brain tumors in the last 20 years is increasing in all ages; however, it has grown more than 40 percent in adult person.

According to clinical presentation among the studied 50 patients; the most common clinical manifestation was 
headache which was found in 15 patients $(30 \%) .10$ patients had disturbed conscious level (20\%), 12 patients had hemiparesis $(24 \%)$ and 8 patients had convulsions $(16 \%)$. M. Afanasjeva [6] found in his study that the most common clinical symptom recorded (in isolation or combined) were headache ( in 67 patients $(51.4 \%$ ) of the cases; it was recorded as a single symptom in 23 patients $(34.3 \%)$ of the cases. M. Ozawa [7], found in a similar study that included 8184 cases and 28110 controls that seizure, weakness and confusion had relatively higher predictive values than many other symptoms. Headache on its own was a weak predictor but this was enhanced when combined with other symptoms especially in younger patients.

The study included different pathological types of brain tumors, the most common diagnosis were gliomas with their different grades. S. Lapointe [8] and T. Mamiya [9] found that the most common malignant primary brain tumors in adults are gliomas, T. Mamiya [9] also reported that glioma is the most common primary malignant brain tumor which is comprising approximately $30 \%$ of all primary brain tumors.

As regard the relation between nature of the lesions and tract affection, fiber tracts involvement were classified into four categories (edema, displacement, infiltration and disruption) by DTI mapping and fiber tractography, This classification of tract involvement was similar to the studies done by Field [10]. However, this was slightly different from the classification done by $\mathrm{Yu}$ [2] who divided the relationship between tumor and tracts into three types only; type I: simple displacement, type II: displacement with disruption, and type III: simple disruption.

We had classified brain tumors in this study into benign and malignant groups, the study included 50 patients 44 of them have malignant lesions $(88 \%)$ of the cases and 6 have benign lesions $(12 \%)$ of the cases, we found that there was a statistical difference between the two groups, with prevalence of displacement among the benign group and infiltration and disruption among the malignant group with $\mathrm{P}$ value $<0.05$. This in contrary to the effect of edema where there was no significant difference between the two groups, with $\mathrm{P}$ value $>0.05$. that agreed with study done by G. Karthika [11] who found in their study on 30 patients with brain tumors that high grade gliomas were significantly associated with infiltration of the white matter tracts and with S. Panesar [12 ]who reported in their study that meningiomas, tend to displace white matter rather than specifically damage or destroy it while high-grade gliomas, comprised of aberrant neural tissue, may infiltrate white matter tracts, causing a combination of displacement, disruption, or outright destruction of white matter and we agreed with the result of the study of V. Itagiba [13] who found that the displacement pattern was seen in all the types of tumor. Disruption and infiltration patterns were found in glioblastomas multiforme and anaplastic astrocytomas. A. Dubey [14] in their study on 34 patients with brain tumors they reported that white matter tracts may be pathologically altered by the tumor in several ways. They may be displaced, infiltrated by tumor and/or edema, or destroyed. Unfortunately, these alterations are not exclusive in a given tumor or even in a given white matter tract.

Three hundred white matter tracts were studied in 50 patients in this study and we found that the most common tract that was affected was the Cortico-Spinal Tract that was affected in 39 cases then Superior Longitudinal Fasiculus that was affected in 38 cases, G. Karthika [11] observed that in their study that the Cortico-Spinal tract was the most common tract to be affected.

Quantitative analysis of the fiber tracts was obtained by measuring the FA values of each affected tract, with comparison with the contra lateral homologous tracts in the unaffected hemisphere as regard to the following tracts, Cortico-Spinal Tract, Superior and Inferior Longitudinal Fasciculus tract, Cingulum, Middle Cerebellar Peduncle and Corpus Callosum and we found that there was significant difference of values of fractional anisotropy (FA) between the affected tracts and the contralateral unaffected tracts in all above mentioned tracts, that agreed with Beppu. T [15] in his study that found that the mean FA value significantly differed between normal brain regions and glioblastoma lesions and also agreed with Kallenberg, K. [16] who found that there was a significant Fractional anisotropy reduction in the tumor corresponding region of the Corpus Callosum of both patient groups compared to controls.

In this study 45 patients with different pathological types of brain tumors were assessed by motor functional MRI, 5 patients were excluded as they were non cooperative patients. Localization of the primary motor hand area with functional MRI was done, The distance relation of primary motor hand area to the tumor was classified according to the distance between it and the tumor border into:(a) primary hand motor area was more than $2 \mathrm{~cm}$ away from the tumor that was reported in 19 patients $(42.22 \%)$, (b) primary hand motor area was 1-2 cm away from the tumor was reported in 5 patients and (c) primary hand motor area was less than $1 \mathrm{~cm}$ away from the tumor was reported in 5 patients $(11.11 \%)$, (d) primary hand motor area is zero distance from the tumor border or lies within the tumor and/or infiltrated by it was reported in 16 patients $(35.55 \%)$.

After analysis of both DTI tractography and motor functional MRI data, the neurosurgeons were asked to assess, the type of surgical approach and the extent of surgical resection established with conventional MR images and with the same images integrated with the tractography and motor functional MRI.

Treatment plans before and after DTI \& functional MR imaging differed in in 10 patients $(22.2 \%)$ of cases as regard to surgical approach and in 28 patients $(62.2 \%)$ of cases as regard to modification of extent of resection with significant relation ( $\mathrm{P}<.05)$, in the previous studies of Romano [17] who found that the utilization of DTI/fMRI in corticotomy led to a change of the surgical approach for resection in $16 \%-21 \%$ of cases. More recent studies by Cao [18] "who found in his retrospective analysis that preoperative planning using DTI enabled the surgeon to investigate the specific surgical approach for each individual patient so it can significantly 
improve the surgical outcome, he had also concluded in his study that preoperative planning using DTI improves neurologic outcome in patients with brainstem lesions. Preoperative estimation of damage was shown to be possible using fMRI/DTI data in a recent study by Castellano [19] showed that the extent of resection and thus, prognosis can be predicted by DTI. In his study, tumors that were infiltrating the white matter tracts were less likely to be resected completely in comparison to tumors in which white matter tracts were intact.

G. Karthika1 [11] reported that MR-tractography provides the neurosurgeon with a new anatomical view that can change the surgical resection planning of a cerebral mass in their study this technique modified the surgical approach to corticotomy in $(21 \%)$ of cases, permitted the definition of the resection margins in (64\%) of cases and resulted in an overall modification of the procedure in $82 \%$ of cases. He had reported in his study that DTI is a very important tool for the preoperative planning of surgery.

Krings [20] and Hoeller [21] in their study that investigated more than 100 patients concluded that a combination of MRI and fMRI played a major role in improving postoperative outcomes of patients and recommended that pre-surgical fMRI should be included in preoperative neuroimaging protocols.

\section{Conclusion}

1) Diffusion tensor imaging represents the only technique that is able to visualize in vivo white-matter tracts compatible with cerebral myelinated fibers, so that it can provide a great help in pre-operative neurosurgical planning.

2) The employment of neuro-radiological techniques (including fMRI and MR-tractography) was proved to be useful in the definition of the relationship between the tumor and adjacent areas of cortical function.

3) The localization of some white matter fiber tracts with respect to a cerebral tumor is of vital importance in neurosurgical planning, for both the identification of the access site and the definition of resection margins.

4) The neurosurgical intervention in a cerebral tumor should balance the maximum surgical resection on one side and the maximum preservation of function on the other. Extensive resection of the lesion leads to reduction of the possible risk of recurrences and achieves better results than radio/chemotherapy. On the other hand, the saving of "functionally relevant" brain areas such as (motor, visual and language functions) improves the quality of life of these patients.

\section{Recommendations}

Pre-surgical DTI tractography and fMRI should be included in standard preoperative neuroimaging for facilitating the planning and performance of functionpreserving operations.

\section{Abbreviation}

FMRI $=$ Functional magnetic resonance imaging, MRI= Magnetic resonance imaging, DTI= Diffusion tensor imaging, $\mathrm{WM}=$ White matter, $\mathrm{cMR}=$ Conventional magnetic resonance imaging, $\mathrm{FA}=$ Fractional anisotropy, $\mathrm{ADC}=$ Apparent diffusion coefficient map.

\section{References}

[1] Commowick O, Fillard P, Clatz O. Detection of DTI white matter abnormalities in multiple sclerosis patients. Warfield Med. Image Comput. Assist. Interv. 2008; 11 (Pt 1): 975982.

[2] Yu HJ, Christodoulou C, Bhise V, et al. Multiple white matter tract abnormalities underlie cognitive impairment in RRMS. Neuroimage. 2012; 59: 3713-3722.

[3] Mori S, van Zijl PC. Fiber tracking: principles and strategies-a technical review. NMR. Biomed. 2002; 15: 468-480.

[4] Farmanfarma KK, Mohammadian M, Shahabinia Z, et al. Brain cancer in the world: an epidemiological review. world cancer Res J. 2019; 5 (4): 1-5. 94.

[5] Sun T, Plutynski A, Ward S, et al. An integrative view on sex differences in brain tumors. Cell Mol Life Sci. 2015; 72 (17): 3323-3342.

[6] Afanasjeva, B, Afanasjevas, D; Jaškevičienè, V; et al. Clinical symptoms of benign brain tumours. Neurol Semin. 2018; 22 (3): 269-271.

[7] Ozawa M, Brennan PM, Zienius K, et al. The usefulness of symptoms alone or combined for general practitioners in considering the diagnosis of a brain tumour: A case-control study using the clinical practice research database (CPRD) (2000-2014). BMJ Open. 2019; 9 (8). https://doi.org/10.1136/bmjopen-2019-029686.

[8] Lapointe S, Perry A and Butowski NA. Primary brain tumours in adults. Lancet. 2018; 392 (10145): 432-446.

[9] Mamiya T, Shimato S, Nishizawa T, et al. Malignant Glioma in the Cerebellum Presenting as Multiple Small Lesions. Case Rep Oncol Med. 2019; 2019: 1-5.

[10] Field AS, Alexander AL, Wu YC, et al. Diffusion tensor eigenvector directional color imaging patterns in the evaluation of cerebral white matter tracts altered by tumor. J Magn Reson Imaging. 2004; 20 (4): 555-562.

[11] G. Karthika, Anand. R and Vayshak K. V. SS. A retrospective study on role of DTI in brain tumours. Int J Contemp Med Surg Radiol. 2019; 4 (4): 14-18.

[12] Panesar SS, Abhinav K, Yeh FC, et al. Tractography for Surgical Neuro-Oncology Planning: Towards a Gold Standard. Neurotherapeutics. 2019; 16 (1): 36-51.

[13] Itagiba VGA, Borges R, da Cruz LCH, et al. Use of diffusion tensor magnetic resonance imaging in the assessment of patterns of white matter involvement in patients with brain tumors: is it useful in the differential diagnosis?. Radiol Bras. 2010;43 (6): 362-368. 
[14] Dubey A, Kataria R and Sinha V. Role of diffusion tensor imaging in brain tumor surgery. Asian J Neurosurg. 2018; 13 (2): 302 .

[15] Beppu T, Inoue T, Shibata Y, et al Fractional anisotropy value by diffusion tensor magnetic resonance imaging as a predictor of cell density and proliferation activity of glioblastomas. Surg Neurol. 2005; 63 (1): 56-61.

[16] Kallenberg K, Goldmann T, Menke J, et al. Glioma infiltration of the corpus callosum: Early signs detected by DTI. J Neurooncol. 2013; 112 (2): 217-222.

[17] Romano A, D’Andrea G, Minniti G, et al. Pre-surgical planning and MR-tractography utility in brain tumour resection. Ekartur Radiol. 2009; 19 (12): 2798-808.

[18] Cao ZK, Lv JP, Wei X, et al. Appliance of preoperative diffusion tensor imaging and fiber tractography in patients with brainstem lesions. Neurol India. 2010; 58 (6): 886-890.

[19] Castellano A, Bello L, Michelozzi C, et al. Role of diffusion tensor magnetic resonance tractography in predicting the extent of resection in glioma surgery. Neuro Oncol. 2012; 14 (2): 192-202.

[20] Krings T, Reinges MHT, Erberich S, et al. Functional MRI for presurgical planning: Problems, artefacts, and solution strategies. J Neurol Neurosurg Psychiatry. 2001;70 (6): 749 760 .

[21] Hoeller M, Krings T, Reinges MHT, et al. Movement artefacts and MR BOLD signal increase during different paradigms for mapping the sensorimotor cortex. Acta Neurochir (Wien). 2002; 144 (3): 279-284. 